\title{
Evaluation the added transverse planes in normal fetal upper mediastinum by new color imaging technique in fetal echocardiography
}

\author{
Yu Qi ${ }^{1}$, Yu Wang ${ }^{2}$, Xue Sun ${ }^{2}$, huiyu Tang ${ }^{1}$, Dong Wang ${ }^{1}$, and Ying Zhang ${ }^{2}$ \\ ${ }^{1}$ Affiliation not available \\ ${ }^{2}$ Shengjing Hospital of China Medical University
}

September 10, 2020

\begin{abstract}
Abstract Introduction: The current study aimed to assess the efficacy of a new scanning procedure in fetal cardiac screening by 6 planes in the upper mediastinum. Methods: This is a prospective study. 123 normal fetuses without extracardiac abnormalities or CHD were enrolled in this study totally. Two sonographers with similar experience were trained for one month about how to obtain the added planes. Sonographer A used Color Doppler combined with the radiant flow (Color-R-flow) while sonographer B used high definition flow imaging combined with the radiant flow (HD-R-flow) to display 6 planes in the upper mediastinum. The difference between these two color modalities in subgroups of different fetal positions (supine, prone, and lateral position) was also discussed. Results: HD-R-flow has higher detection rates of the 6 planes than Color-R-flow. The differences presenting for the left innominate vein and azygos vein (LIV and AzV) view, bilateral subclavian arteries (BSA) view, and the bilateral internal thoracic arteries (BITA) view were significant $(\mathrm{P}<0.01)$. The results also demonstrated that HD-R-flow showed significantly higher detection rates than the Color-R-flow for the LIV and AzV view and the BITA view of the fetuses in the supine and lateral position, and BSA view of fetuses in lateral position. Conclusion: The current study introduced an enhanced scanning procedure to assess vessels in the fetal upper mediastinum. A new color-imaging technique was used to better show tiny vessels. This has confirmed its value in showing a satisfactory detection rate for the 6 cardiac planes.
\end{abstract}

\section{Introduction}

Congenital heart disease (CHD), with an estimated incidence rate of $1 \%$ of newborns, remains to be the most common congenital malformations leading to high morbidity and mortality of neonates [1-3]. Echocardiography is the most effective modality to diagnose CHDs prenatally, which assures proper perinatal management and thus improves the outcome. Theoretically, most CHDs could be detected in the uterus by an experienced fetal echocardiographer [4]. They are sensitive to find subtle changes in morphology and hemodynamics in fetal heart and to trace the clues to make an accurate diagnosis [5]. However, this beyond the ability of most screening sonographers as it needs much experience and expertise. A systematic scanning procedure with standard cardiac planes is in need.

As recommended by ISUOG guidelines for fetal echocardiography, a series of transverse planes is proposed in routine cardiac scans, including the four-chamber view $(4 \mathrm{CV})$, bilateral outflow tracts views, and the three-vessel trachea (3VT) view [6]. Previous reports had suggested a high efficacy in detecting fetal CHDs using these transverse planes [7-10]. However, some abnormalities in the fetal upper mediastinum are apt to be missed by routine cardiac screenings. It is possible to improve the detection of fetal CHDs by including more transverse cardiac planes in addition to the existing recommended views. 
Fetal great vessels could be visualized directly by gray-scale ultrasonography. Color Doppler could undoubtedly enhance morphological information and determine the blood direction. High-definition flow (HD-flow) imaging is a special bidirectional Doppler technique that has the potential benefits of better axial and lateral resolution, fewer blooming artifacts, and improved sensitivity to display vessel perfusion and blood flow continuity than traditional Color Doppler [11-13]. Recently, the use of radiant flow (R-flow) imaging technique is a great improvement in fetal cardiac imaging. By specific algorithm, the index of erythrocyte density in a certain area is converted into height index and then superimposed on the initial coding of color or HD-flow. The flow is then displayed in a sense of depth, resembling a three-dimensional visual effect. Besides, the use of Color Doppler/HD-flow together with the R-flow technique could apparently reduce color overflow and thus show vessels with sharp edges.

The current study aimed to evaluate the efficacy of a detailed procedure exploring vessels in the fetal upper mediastinum. Two color imaging modalities were used to compare their performance in the investigation of these planes.

\section{Materials and Methods}

\section{Study population}

The study population was randomly selected in the pregnant women presented to our center from July 2018 to December 2019. Fetuses with confirmed CHD or extracardiac abnormalities were excluded. Mothers with gestational anemia, diabetes, hypertension, and other high-risk factors were also excluded. All fetuses involved were singletons. In total, 123 pregnant women were enrolled in this study. Maternal gestation age ranged from 20 to 37 weeks. Informed consent was obtained from each patient and the study was approved by the Ethics Committee of Shengjing Hospital of China Medical University. All cases underwent a month postnatal echocardiography follow-up.

\section{Ultrasonography technique}

An ultrasound system (Voluson E10, GE Healthcare, Kretztechnik, Zipf, Austria), equipped with a 2 to $9 \mathrm{MHz}$ transabdominal transducer was used in this study. Each patient was scanned by two screening sonographers with one year of experience, independently, using gray-scale ultrasound and different color techniques. They were both adept at the traditional procedures of fetal cardiac transverse scanning using the $4 \mathrm{CV}$, the left and right outflow tracts view, and the $3 \mathrm{VT}$ view. Then, they were both trained for three months to become familiar with scanning the added planes included in the current study. The specific scanning procedures are achieved by continuously moving the sound beam cephalad along the vertical axis of the fetal thorax. When scanning the root of the great arteries, the sound beam sections through the long-axis of the ductus arteriosus and the right pulmonary artery, together with the short-axis of the aorta and superior vena cava. It is the $3 \mathrm{VV}$. Slight rotating the sound beam to the left, the bifurcation of the pulmonary artery should be demonstrated to show the normal pulmonary artery branching patterns. It is the branch of pulmonary artery bifurcation (BPAB) view. When the beam sections through the arch, the ductus arteriosus, and their convergence, the $3 \mathrm{VT}$ view is obtained. When continuing elevating the sound beam, it demonstrates that the superior vena cava is joined by both the left innominate vein and the posteroanterior drainage of the azygos vein. When the beam is turned further cephalad, it sections across the transverse aortic arch forming a sausage-like structure [14]. The origin of bilateral subclavian arteries (BSA) could be identified in this plane. Scanning around the BSA, the origin of bilateral internal thoracic arteries (BITA) from the ipsilateral subclavian artery can be demonstrated [15]. The related anatomical structures that should be recognized in each plane were listed in Table 1.

For both sonographers involved in the study, the first recognized anatomical structures (including the course of the great vessels) by gray-scale ultrasound. Then, different color-imaging techniques were used to assess the vessels in detail, including the course and flow direction. For sonographer A, he used Color Doppler combined with the radiant flow (Color-R-flow modality) to show the vessels if needed, while HD-flow combined with the radiant flow (HD-R-flow modality) was used by sonographer B when scanning these planes. For both modalities, the scale was set to $20-30 \mathrm{~cm} / \mathrm{s}$ and $40-50 \mathrm{~cm} / \mathrm{s}$ when showing the veins and arteries respectively. 
The number of planes visualized was recorded for each sonographer to make a comparison.

As cardiac apex orientation might pose some effects on the detection of the cardiac views, we also made comparisons among different fetal positions when necessary. Three subgroups were made according to the supine position (cardiac apical pointing to 10-2 o'clock), the prone position (cardiac apical pointing to 4-8 o'clock), and the lateral position (cardiac apical pointing to 2-4 and 8-10 o'clock).

Statistical analysis

The detection rates for the two modalities were compared via McNemar analysis. $P$-values $<0.05$ were considered statistically significant. All statistical analyses were performed using commercially available software (SPSS, release 17.0).

\section{Results}

The feasibility of the algorithm was studied in 123 fetuses. The mean GA was $26 \pm 2.3$ weeks and the mean BMI was $25 \pm 3.6 \mathrm{~kg} / \mathrm{m}^{2}$. In total, 6 cardiac planes were included in the current study. The detection rate for each plane is presented in Table 2 . The $3 \mathrm{VV}$ and $3 \mathrm{VT}$ view are in high visualization rate, varying from $88.62 \%$ to $97.56 \%$, for the two color imaging modalities. HD-R-flow modality showed a higher detection rate for all the 6 planes than the Color-R-flow method, with significance presenting for the left innominate and azygos vein (LIV and AzV) view ( $71.54 \%$ vs. $35.77 \%$ ), bilateral subclavian arteries (BSA) view (85.37\% vs. $60.16 \%$ ), and the bilateral internal thoracic arteries (BITA) view (75.61\% vs. $50.41 \%$ ) (Figure).

As Doppler shift could be affected by the angle between the sound beam and the flow direction, we speculated that the detection of these cardiac planes would also be affected by different fetal positions. Since the differences of detection rates of $3 \mathrm{VV}, \mathrm{BPAB}$ and $3 \mathrm{VT}$ views were of no significance, the affection of the fetal position was not under discussion. Then, fetuses were sub-grouped according to different cardiac orientations and comparisons were made between the two color-imaging modalities for the detection of the last three planes. The results demonstrated that HD-R-flow modality showed a significantly higher detection rate than the Color-R-flow modality for the LIV and AzV view (Supine and Lateral position), BSA view (Lateral position), and the BITA view (Supine and Lateral position). However, for the prone position, the detection for each plane was very low and no significance was demonstrated for each of the two modalities (Table 3).

\section{Discussion}

The detection of CHD remains a great challenge during routine obstetric screenings. It is realized that some severe CHDs could be identified by fetal echocardiography early in the 1990s [16-17]. Initially, only the 4CV was used to determine whether a CHD was in existence in the circumstance of asymmetrical chambers, large-in-sized VSD/AVSD, or space-occupying lesions [18-20]. Obvious limitations had been shown for this modality as it could only identify anomalies at the atrial and ventricular level. The situation had been improved impressively when the left and right outflow tract views were included in the routine diagnostic procedures besides the $4 \mathrm{CV}$, which was supported by the fact of a significantly higher detection rate for conotruncal anomalies [19, 21-23].

Recently, the new fetal echocardiography guideline had shown its advantage in detecting more than $90 \%$ CHDs in the uterus, as documented by several teams and regional studies. The addition of the 3VT view apparently contributes to the improved performance in this new guideline [24]. It was a great development to evaluate fetal great arteries anatomy as the characteristic sonograms in this specific plane are apt to recognize by general examiners. The ductus arteriosus, aortic arch, and their convergence in a "V" configuration could be visualized on the left side of the trachea. In fact, most arch anomalies could be demonstrated by this plane, such as double aortic arch, right aortic arch, aberrant left/right subclavian artery, etc [25-26]. We proved a very high detection rate for this plane by screening the sonographers of one-year experience.

Unfortunately, many screening sonographers concern much on the $3 \mathrm{VT}$ view while anatomical structures between the level of the outflow tracts and 3VT planes are not noticed. When gradually elevating the sound beam from the outflow tracts views, it firstly sections through the pulmonary trunk and its branches, and then 
the convergence of the arch and the ductus arteriosus. We previously reported that it is crucial to searching around the $3 \mathrm{VV}$ to exclude malformations associated with the aortic origin of the left/right pulmonary artery [27]. Also, in the case of the pulmonary sling, the abnormal origin together with the retroesophageal course of the left pulmonary artery from the right pulmonary artery could only be shown at the 3VV while it could not be shown at the $3 \mathrm{VT}$ view. We added these 2 cardiac planes in the current study to explore the feasibility in routine screenings. To our point, the $3 \mathrm{VV}$ is relatively easy to obtain while it is very difficult to show the BPAB view. A learning curve is needed for scanning. The pulmonary branching patterns could better be identified by gray-scale ultrasound when searching along the pulmonary trunk, as far as our experience is concerned. Though the addition of color imaging helps to determine the flow direction of the vessels, it does not seem to help much in improving the detection of the branching when searching along the pulmonary trunk.

However, color techniques are necessary to investigate small or tiny vessels, as the vascular wall is too thin to be visualized by gray-scale ultrasound. Color Doppler has long been used in echocardiography while it cannot show vessels with a low velocity with a good effect. Doppler shift can be immensely affected by the angle of the sound beam which contributes to the low sensitivity in showing small vessels with low velocity. The introduction of HD-flow is a great innovation in fetal color imaging. It shows a higher sensitivity to display blood flow in better perfusion than the traditional Color Doppler technique [11-13]. However, excessively higher sensitivity may lead to color overflow and thus decrease the spatial resolution of the vessels. The R-flow technique could display color flow in a three-dimensional effect by adding an algorithm upon initially Color/HD-flow. When using HD-R-flow, the vessels can then be displayed with both high sensitivity and high resolution with a sharp edge.

Technical renovations can further promote the launch of a new and improved protocol for fetal cardiac scanning. Aorta, the pulmonary artery, and the superior vena cava are the three great vessels which should be scanned according to the current guidelines [6]. Other smaller vessels are existing in the upper mediastinum, such as the tributaries of superior vena cava (SVC), branches of the aortic arch (i.e. left/right carotid artery, left/right subclavian artery), and the internal thoracic artery. We tried to explore these vessels in routine cardiac scans and thus to screen more malformations in potential. The application of the new color technique makes it possible to approach the skill for the detection of these additional vessels with small size and low-velocity.

Three planes above the level of 3VT view were added in the current study. Anatomically, the right innominate vein goes down straight and continues to be the SVC, when the LIV joins it. AzV is a small-sized vessel that serves as a little tributary of SVC. It is hard to be identified using Color Doppler in the past while it is relatively easy to show in many fetuses when scanned by HD-flow. In the case of supracardiac anomalous pulmonary venous connect, the flow of pulmonary veins may drain into the LIV via the vertical vein. The examiner may find the indirect sign of a dilated LIV and thus to trace its origin to make a diagnosis [28]. In other cases, when the LIV is not shown on this plane, the examiner should consider the possibility of a left $\mathrm{SVC}$. If a dilated $\mathrm{AzV}$ is in visualization in this plane, the possibility of an interrupted inferior vena cava might be in consideration [29].

For the BSA plane, it is useful to make the diagnosis of an aberrant left/right subclavian artery, in which the bilateral subclavian arteries arise from almost the same position at the proximal descending aorta. This is very different from normal fetuses, in which the origins of the left and right subclavian artery were located at the two ends of the arch. At $3 \mathrm{VT}$ view, AzV sometimes may be misrecognized as an aberrant right subclavian artery. The scanning of the BSA plane helps to make a differential diagnosis. In addition, the size of the thymus could be assessed by the BITA plane since it is surrounded by BITA. A small-sized or disappeared thymus at this plane may suggest the possibility of Digeorge Syndrome [30].

At last, we want to comment on the efficacy of the detailed scanning procedure performed by the screening sonographers. HD-R-flow apparently showed a better performance than color-R-flow in showing small vessels in the three planes above the 3VT level. The difference between the two color-imaging modalities may be related to different degrees of influence by Doppler shift as the difference presents only for fetuses with 
apical/lateral insonation. BRAVO et al. reported a higher detection rate for BSA and BITA plane than our results [31]. An echocardiographer apparently has much more experience than a screening sonographer with only one-year experience. However, we can expect a long term training session may improve the detection of these added planes. For all the 6 planes involved in the current study, the detection rate is above $70 \%$, suggesting a potential wide clinical usage in the future.

\section{Study limitation}

The study was limited in that it was relatively small research and did not recruit CHD fetuses. We expect to carry out a multi-center study in a normal screening population to evaluate the sensitivity and specificity of this detailed scanning procedure in cardiac screening. Besides, to our acknowledgment, the study about the R-flow on fetal echocardiography is rare. We expect the advanced application of R-flow on the prenatal diagnosis of the fetal heart.

\section{Conclusions}

The current study introduced an enhanced scanning procedure to assess vessels in the fetal upper mediastinum. New color-imaging techniques were used to better show tiny vessels with low-velocity. A short-term training session made it possible for the screening sonographers to become familiar with the new scanning procedure; this has confirmed its value in showing a satisfactory detection rate for the 6 cardiac planes.

\section{Acknowledgements}

We thank for all the medical specialists who helped collecting the experimental data.

\section{Consent for publication}

Not applicable.

\section{Competing interests}

The authors declare that they have no competing interests.

\section{References:}

1. Hoffman JI, Kaplan S: The incidence of congenital heart disease. J Am Coll Cardiol 2002:39(12):1890-1900.

2. Massaro AN, El-Dib M, Glass P, et al: Factors associated with adverse neurodevelopmental outcomes in infants with congenital heart disease. Brain Dev 2008:30(7):437-446.

3. Cuneo BF, Curran LF, Davis N, et al: Trends in prenatal diagnosis of critical cardiac defects in an integrated obstetric and pediatric cardiac imaging center. J Perinatol 2004:24(11):674-678.

4. Ayres NA: Advances in fetal echocardiography. Tex Heart Inst J 1997: 24(4):250-259.

5. Zhang Y, Cai AL, Ren WD, et al: Identification of fetal cardiac anatomy and hemodynamics: a novel enhanced screening protocol. BMC Pregnancy Childbirth 2016:16:145.

6. Carvalho JS, Allan LD, Chaoui R, et al: ISUOG practice guidelines (updated): sonographic screening examination of the fetal heart. Ultrasound Obstet Gynecol 2013: 41(3): 348-359.

7. Bravo C, Gámez F, Pérez R, et al: Fetal Aortic Arch Anomalies: Key Sonographic Views for Their Differential Diagnosis and Clinical Implications Using the Cardiovascular System Sonographic Evaluation Protocol [published online ahead of print Jun 35, 2016]. J Ultrasound Med 2016:35(2):237-251.

8. Yang ZJ, Pei QY, Li YT, et al: Continuous transverse scanning of the fetal heart using a cross-sectional image database of common fetal congenital heart deformities. Taiwan J Obstet Gynecol 2016:55(2):176-182.

9. Weissmann-Brenner A, Zajicek M, Weisz B, et al: Feasibility of detection of the 3-vessel and trachea view using 3-dimensional sonographic volumes. J Ultrasound Med 2014:33(4):681-685. 
10. Li H, Wei J, Ma Y, et al: Prenatal diagnosis of congenital fetal heart abnormalities and clinical analysis. J Zhejiang Univ Sci B 2005:6(9):903-906.

11. He YH, Liu K, Gu XY, et al: The application of high definition flow imaging in fetal hemodynamics. Clin Exp Obstet Gynecol 2015: 42(1):11-17.

12. Hata T, Dai SY, Inubashiri E, et al: Four-dimensional sonography with B-flow imaging and spatiotemporal image correlation for visualization of the fetal heart. J Clin Ultrasound 2008: 36(4):204-207.

13. Rizzo G, Pietrolucci ME, Aiello E, et al: Are there any differences in three-dimensional placental vascular indices obtained using conventional power Doppler and high-definition flow imaging? J Matern Fetal Neonatal Med 2012: 25(9):1664-1667.

14. Yoo SJ, Min JY, Lee YH, et al: Fetal sonographic diagnosis of aortic arch anomalies. Ultrasound Obstet Gynecol 2003: 22(5):535-546.

15. Bravo C, Gámez F, Pérez R, et al: Fetal Aortic Arch Anomalies: Key Sonographic Views for Their Differential Diagnosis and Clinical Implications Using the Cardiovascular System Sonographic Evaluation Protocol. J Ultrasound Med 2016:35(2):237-251.

16. Copel JA, Pilu G, Green J, et al: Fetal echocardiographic screening for congenital heart disease: the importance of the four-chamber view. Am J Obstet Gynecol 1987: 157(3):648-655.

17. Allan LD, Crawford DC, Chita SK, et al: Prenatal screening for congenital heart disease. Br Med J (Clin Res Ed) 1986: 292(6537):1717-1719.

18. Shi C, Song L, Li Y, et al: Value of four-chamber view of the fetal echocardiography for the prenatal diagnosis of congenital heart dise. Zhonghua Fu Chan Ke Za Zhi 2002:37(7):385-387.

19. Sharland G. Routine fetal cardiac screening: what are we doing and what should we do? Prenat Diag 2004:24(13): 1123-1129.

20. Sharland GK, Allan LD. Screening for congenital heart disease prenatally. Results of a $21 / 2$-year study in the South East Thames Region. Br J Obstet Gynaecol 1992: 99(3): 220-225.

21. Hernandez-Andrade E, Patwardhan M, Cruz-Lemini M, et al: Early Evaluation of the Fetal Heart. Fetal Diagn Ther 2017:42(3):161-173.

22. Kusajima K, Hoashi T, Kagisaki K, et al: Reoperative double ventricular outflow tract reconstruction in grown-up congenital heart disease patients with conotruncal anomalies. Gen Thorac Cardiovasc Surg 2015:63(11):595-600.

23. Quartermain MD, Glatz AC, Goldberg DJ, et al: Pulmonary outflow tract obstruction in fetuses with complex congenital heart disease: predicting the need for neonatal intervention. Ultrasound Obstet Gynecol 2013:41(1):47-53.

24. Viñals F, Heredia F, Giuliano A. The role of the three vessels and trachea view (3VT) in the diagnosis of congenital heart defects. Ultrasound Obstet Gynecol 2003:22(4):358-367.

25. Wang Y, Fan M, Siddiqui FA, et al: Strategies for Accurate Diagnosis of Fetal Aortic Arch Anomalies: Benefits of Three-Dimensional Sonography With Spatiotemporal Image Correlation and a Novel Algorithm for Volume Analysis. J Am Soc Echocardiogr 2018:31(11):1238-1251.

26. Yagel S, Arbel R, Anteby EY, et al: The three vessels and trachea view (3VT) in fetal cardiac scanning. Ultrasound Obstet Gynecol 2002:20(4):340-345.

27. Ma J, Zhang Y, Wang Y, et al: Prenatal two-and three-dimensional echocardiographic diagnosis of anomalous origin of one pulmonary artery from the ascending aorta: Case report and literature review. J Clin Ultrasound 2020:23. 
28. Sun X, Zhang Y, Fan M, et al: Role of four-dimensional echocardiography with high-definition flow imaging and spatiotemporal image correlation in detecting fetal pulmonary veins. Echocardiography 2017:34(6):906-914.

29. Xu Q, Sun HX, Xie JS, et al: Zhonghua Fu Chan Ke Za Zhi 2018:53(3):149-154.

30. Tramontana A, Hartmann B, Hafner E. DiGeorge syndrome chromosome region deletion and duplication: Prenatal genotype-phenotype variability in fetal ultrasound and MRI. Prenat Diagn 2019:39(13):1225-1234.

31. Bravo C, Gámez F, Pérez R, et al: Fetal Aortic Arch Anomalies: Key Sonographic Views for Their Differential Diagnosis and Clinical Implications Using the Cardiovascular System Sonographic Evaluation Protocol. J Ultrasound Med 2016:35(2):237-251.

Tables:

Table 1 Anatomical structures recognized at each view of the added planes in the current study

\begin{tabular}{ll}
\hline Cardiac planes & Anatomical structures \\
\hline 3VV & The long-axis of MPA, RPA and DA, the short-axis of AAO, SVC and DAO. \\
BPAB & MPA, the bifurcation into the RPA and LPA and the short-axis of AAO, DAO and SVC. \\
3VT & The V-shape formed by DA and AAO, the short-axis of SVC and DAO, trachea. \\
LIV and AzV & SVC is joined by both the LIV and the posteroanterior drainage of AzV. \\
BSA & The S-shaped formed by BSA. \\
BITA & The thymus is located in the middle of the chest and laterally by BITA. \\
\hline
\end{tabular}

3VT, three-vessel trachea view; 3VV, three-vessel view; AAO, aortic arch; AzV: azygos vein; BITA: bilateral internal thoracic arteries; BPAB: branch pulmonary artery bifurcation; BSA: bilateral subclavian arteries; DA, ductus arteriosus; DAO, descending aorta; LIV: left innominate vein; SVC, superior vena cava.

Table 2 The detection rates for six cardiac planes in normal fetal upper mediastinum using two color-imaging modalities

\begin{tabular}{llll}
\hline Cardiac planes & HD-Radiant flow n $(\%)$ & Color-Radiant flow n $(\%)$ & P-value \\
\hline 3VV & $111(90.24)$ & $109(88.62)$ & 0.500 \\
BPAB & $87(70.73)$ & $84(68.29)$ & 0.250 \\
3VT & $120(97.56)$ & $119(96.75)$ & 1.000 \\
LIV and AzV & $88(71.54)$ & $44(35.77)$ & $<0.01(0.000)$ \\
BSA & $105(85.37)$ & $74(60.16)$ & $<0.01(0.000)$ \\
BITA & $93(75.61)$ & $62(50.41)$ & $<0.01(0.000)$ \\
\hline
\end{tabular}

3VT, three-vessel trachea view; 3VV, three-vessel view; AzV, azygos vein; BITA, bilateral internal thoracic artery; BPAB, Branch pulmonary artery bifurcation; BSA, bilateral subclavian artery; LIV, left innominate vein.

Table 3 The detection of the three cardiac planes above the three-vessel trachea view according to different fetal positions

\begin{tabular}{|c|c|c|c|c|c|c|c|c|c|c|}
\hline $\begin{array}{l}\text { Cardiac } \\
\text { planes }\end{array}$ & Supine & Supine & Supine & Supine & Lateral & Lateral & Lateral & Prone & Prone & Prone \\
\hline & $\begin{array}{l}\text { HDFI n } \\
(\%)\end{array}$ & $\begin{array}{l}\text { CDFI n } \\
(\%)\end{array}$ & P-value & $\begin{array}{l}\text { HDFI n } \\
(\%)\end{array}$ & $\begin{array}{l}\text { HDFI n } \\
(\%)\end{array}$ & $\begin{array}{l}\text { CDFI n } \\
(\%)\end{array}$ & P-value & $\mathrm{P}$-value & $\begin{array}{l}\text { HDFI n } \\
(\%)\end{array}$ & $\begin{array}{l}\text { CDFI r } \\
(\%)\end{array}$ \\
\hline
\end{tabular}




\begin{tabular}{lllllllllll}
\hline $\begin{array}{l}\text { Cardiac } \\
\text { planes }\end{array}$ & Supine & Supine & Supine & Supine & Lateral & Lateral & Lateral & Prone & Prone & Prone \\
\hline LIV and & 26 & 11 & $<0.05$ & 56 & 56 & 31 & $<0.05$ & $<0.05$ & 6 & 2 \\
AzV & $(86.67)$ & $(36.67)$ & $(0.000)$ & $(70.89)$ & $(70.89)$ & $(39.24)$ & $(0.000)$ & $(0.000)$ & $(42.86)$ & $(14.29)$ \\
view & & & & & & & & & & \\
BSA & 27 & 23 & 0.125 & 72 & 72 & 47 & $<0.05$ & $<0.05$ & 6 & 4 \\
view & $(90.00)$ & $(76.67)$ & & $(91.14)$ & $(91.14)$ & $(59.49)$ & $(0.000)$ & $(0.000)$ & $(42.86)$ & $(28.57)$ \\
BITA & 27 & 21 & $<0.05$ & 58 & 58 & 35 & $<0.05$ & $<0.05$ & 8 & 6 \\
view & $(90.00)$ & $(70.00)$ & $(0.031)$ & $(73.42)$ & $(73.42)$ & $(44.30)$ & $(0.000)$ & $(0.000)$ & $(57.14)$ & $(42.86)$ \\
\hline
\end{tabular}

Supine: Indicates fetal cardiac orientation is between 10-2 o'clock

Lateral: Indicates fetal cardiac orientation is between 2-4 and 8-10 o'clock

Prone: Indicates fetal cardiac orientation is between 4-8 o'clock

$\mathrm{AzV}$, azygos vein; BITA: bilateral internal thoracic artery; BSA, bilateral subclavian artery; LIV, left innominate vein.

\section{Figure legends:}

The added planes acquired by Color-R-flow and HD-R-flow in a fetus of 25 week. A. three-vessel view $(3 \mathrm{VV})$, B. branch pulmonary artery bifurcation view (BPAB), C. three-vessel trachea view (3VT), D. the left innominate vein and azygos vein view, E. the bilateral subclavian arteries view, F. the bilateral internal thoracic arteries view.

AA, aortic arch; AO, ascending aorta; AzV, azygos vein; DA, ductus arteriosus; DAO, descending aorta; LITA, left internal thoracic artery; LIV, left innominate vein; LPA, left pulmonary artery; LSA, left subclavian artery; MPA, main pulmonary artery; R-ITA, right internal thoracic artery; RPA, right pulmonary artery; RSA, right subclavian artery; SVC, superior vena cava; T, trachea.

\section{Abbreviations:}

3VT, three-vessel trachea; 3VV, three-vessel vein; 4CV, four chamber view; Azy, azygos vein; BITA, bilateral internal thoracic arteries; BPAB, branch pulmonary artery bifurcation; BSA, bilateral subclavian arteries; Color-R-flow, Color Doppler combined with radiant flow; CHD, congenital heart disease; HD-flow, high definition flow imaging; HD-R-flow; high definition flow imaging combined with radiant flow; LIV, left innominate vein; R-flow, radiant flow; SVC, superior vena cava.

\section{Hosted file}

$\backslash$ begin $\{\mathrm{CJK}\}\{\mathrm{UTF} 8\}\{\mathrm{gbsn}\}$. \end\{CJK\}\selectlanguage\{english\}eps available at https://authorea. } com/users/355685/articles/478823-evaluation-the-added-transverse-planes-in-normal-fetalupper-mediastinum-by-new-color-imaging-technique-in-fetal-echocardiography 